\title{
A novel T wave cancellation method based on MAP estimation for $\mathrm{P}$ wave extraction ${ }^{1}$
}

\author{
Chang-An Wei ${ }^{\mathrm{a}}$ and Huhe Dai ${ }^{\mathrm{b}, *}$ \\ ${ }^{a}$ Automatic Test And Control Institute, Harbin Institute of Technology, Harbin, China \\ ${ }^{\mathrm{b}}$ Shenzhen Institutes of Advanced Academy of Sciences, Chinese Academy of Sciences, China
}

\begin{abstract}
P}$ wave and $\mathrm{T}$ wave in human-body electrocardiogram (ECG) signals often fuse together when atrial premature contract (APC) occurs. $\mathrm{P}$ waves within the fused signals are valuable for the measurement of $\mathrm{P}$ wave parameters as well as diagnosis of supra-ventricular arrhythmias. However, the problem of extracting $\mathrm{P}$ wave from the fused signals is seldom addressed. In this study, a novel $\mathrm{T}$ wave cancellation method for $\mathrm{P}$ wave extraction based on maximum a posteriori (MAP) estimation is proposed. In order to accurately cancel the $\mathrm{T}$ wave within the fused signal, $\mathrm{T}$ wave and the timing point of $\mathrm{T}$ wave peak are estimated simultaneously. The estimated timing point of $\mathrm{T}$ wave peak is used as alignment reference point for $\mathrm{T}$ wave subtraction. Simulation results show that the proposed method outperform the traditional $\mathrm{T}$ wave cancellation method in terms of both normalized mean square error and cross-correlation index. The results for real ECGs with APC demonstrate that the extracted $\mathrm{P}$ waves using the proposed method are more similar to the non-overlapping $\mathrm{P}$ waves in terms of morphology than the ones using the traditional $\mathrm{T}$ wave cancellation method.
\end{abstract}

Keywords: Electrocardiogram, $\mathrm{P}$ wave extraction, atrial premature contract, maximum a posteriori

\section{Introduction}

Human-body electrocardiogram (ECG) is the reflection of the heart's electrical activity, and ECG is of great value for cardiac arrhythmia diagnosis. A typical ECG signal is comprised of $\mathrm{P}$ wave, QRS complex and T wave. $\mathrm{P}$ wave is generated during atrial depolarization, while QRS complex and $\mathrm{T}$ wave are induced by ventricular depolarization and ventricular polarization, respectively. Compared with QRS complex, $\mathrm{P}$ wave is rather weak, and hence is more difficult to deal with [1]. P wave must be firstly detected (i.e., locating the onset, peak and offset points of $\mathrm{P}$ wave), then the related parameters, such as $\mathrm{P}$ wave duration, $\mathrm{P}$ wave amplitude and $\mathrm{P}-\mathrm{R}$ interval, can be measured and the further diagnosis of supra-ventricular arrhythmias can be continued [2-5].

Unfortunately, when atrial premature contractions (APCs) occur, $\mathrm{P}$ wave is often fused with $\mathrm{T}$ wave of the preceding beat (i.e., $\mathrm{P}$ on $\mathrm{T}$ phenomenon) and becomes invisible partly or completely. In order to cal-

\footnotetext{
${ }^{1}$ This work is supported by National Natural Science Foundation of Youth Science Foundation under Grant 31300816, and in part by the Fundamental Research Funds for the Central Universities under Grant HIT.NSRIF.2012014.

*Address for Corresponding author: Dr. Huhe Dai, Shenzhen Institutes of Advanced Academy of Sciences, Chinese Academy of Sciences, 1068 Xueyuan Avenue, Shenzhen University Town, Shenzhen, P.R.China. Tel.:86-755-86392288; Fax:86-75586392299; E-mail:hh.dai@siat.ac.cn.
}

0959-2989/15/\$35.00 @ 2015 - IOS Press and the authors. 
culate the above mentioned parameters in this special case, $\mathrm{P}$ wave must be extracted from the fused signal. And extracting P wave from the fused signal is fundamental to APC analysis. It has been confirmed that APCs are strongly related with the onset of atrial fibrillation. The more frequently APC happens, the higher the risk of initiating atrial fibrillation is [6]. Due to the fusion of $\mathrm{P}$ wave with $\mathrm{T}$ wave, the $\mathrm{P}$ wave within the fused signal is not utilized for APC analysis. There was once a debate whether it was necessary to make measurement when $P$ wave fuses together with T wave. CSE (Common Standards for Quantitative Electrocardiography) Working Party recommended that no measurement should be made, since the measurement is not accurate enough [7]. However, there were some opinions that it was too harsh a rule and programs should at least attempt the measurement and mark the result as unreliable.

In recent years, several works have been done to extract $\mathrm{P}$ wave from the fused $\mathrm{P}$ wave and $\mathrm{T}$ wave. Cabasson et al. [8-10] proposed a time delay estimation technique for P-R interval measurement during exercise or recovery, where the influence of the overlapping T wave on P-R measurement was considered. $\mathrm{T}$ wave was first modeled using polynomial functions or piecewise linear functions, and then maximum likelihood estimator is used to estimate P-R interval. Lin et al. [11] developed a T wave cancellation method for extracting $\mathrm{P}$ wave from the fused signal. The QRS-T template was subtracted from the QRS$\mathrm{T}$ containing overlapping $\mathrm{P}$ wave, and then $\mathrm{P}$ wave was detected in the remaining signal. The QRS-T template in Lin's method is from the preceding normal beat.

In this paper, we focus on extracting $\mathrm{P}$ wave from the fused $\mathrm{P}$ wave and $\mathrm{T}$ wave in rest ECG. Considering that (1) the existing $\mathrm{T}$ wave cancellation methods use $\mathrm{R}$ wave peak as the alignment reference point before subtracting $\mathrm{T}$ wave, which would influence the accuracy of $\mathrm{T}$ wave alignment and hence would lead to strong $T$ wave residues in the remaining signal, (2) in rest ECG, there are a lot of non-overlapping $\mathrm{T}$ waves and $\mathrm{P}$ waves (i.e., the prior information of $\mathrm{T}$ wave and $\mathrm{P}$ wave) which are not fully utilized, and (3) the performance of MAP method for extracting atrial activity (AA) signal from ECG with atrial fibrillation (AF) is satisfactory [12], we develop the MAP based method which could estimate T wave and the timing location of $\mathrm{T}$ wave simultaneously.

\section{Modeling PDFs of $P$ wave and $T$ wave}

In this section, we first introduce the Generalized Gaussian model. Then, the steps of modeling the PDFs of $\mathrm{P}$ wave and $\mathrm{T}$ wave are presented in detail.

\subsection{A generalized gaussian model}

The Generalized Gaussian model is expressed as follows [13]:

$$
\begin{aligned}
& p(z \mid \mu, \sigma, \beta)=\frac{\omega(\beta)}{\sigma} \exp \left[-c(\beta)\left|\frac{z-\mu}{\sigma}\right|^{2 /(1+\beta)}\right] \\
& c(\beta)=\left[\frac{\Gamma[1.5(1+\beta)]}{\Gamma[0.5(1+\beta)]}\right]^{1 /(1+\beta)} \\
& \omega(\beta)=\left[\frac{\Gamma[1.5(1+\beta)]^{0.5}}{(1+\beta) \Gamma[0.5(1+\beta)]^{1.5}}\right]
\end{aligned}
$$


where $\mu$ and $\sigma$ are the mean and standard deviation of variable $z$, respectively, which are estimated using the training data. $\Gamma$ denotes Gamma function. $\beta$ can be determined by a MAP estimation [14]:

$$
\hat{\beta}=\arg \max _{\beta}(\operatorname{Pr}(z \mid \beta))
$$

where $\operatorname{Pr}(t)$ is the probability of variable $t$. The Generalized Gaussian model can describe platykurtic, Gaussian, and leptokurtic distributions by varying $\beta$. The details about the Generalized Gaussian model can refer to [13]. Through Eq.(1), the PDF of a variable can be modeled using parameters $\mu, \sigma$ and $\beta$.

\subsection{Modeling PDFs of $P$ wave and $T$ wave using generalized gaussian model}

In rest ECG, we can obtain non-overlapping $\mathrm{P}$ waves and $\mathrm{T}$ waves in the non-APC beats. These $\mathrm{P}$ waves and $\mathrm{T}$ waves would be used as the training samples for modeling PDFs of $\mathrm{P}$ wave and $\mathrm{T}$ wave, respectively.

In this study, both $\mathrm{P}$ wave and $\mathrm{T}$ wave are treated as vector variables. Let $X=\left[x_{1} \cdots x_{N}\right]$ and $Y=$ $\left[\begin{array}{lll}y_{1} & \cdots & y_{M}\end{array}\right]$ represent $\mathrm{P}$ wave and T wave, respectively. Let $N=N_{b}+N_{a}+1$ and $M=M_{b}+M_{a}+1$, where

$N_{b}$-sample number between the onset and peak of $\mathrm{P}$ wave.

$N_{a}$-sample number between the peak and offset of P wave.

$M_{b}$-sample number between the onset and peak of T wave.

$M_{a}$-sample number between the peak and offset of T wave.

Suppose the training beat number is $\mathrm{K}$, then $N_{b}, N_{a}, M_{b}, M_{a}, \mu, \sigma$ and $\beta$ are determined according to the following steps:

a) $N_{b}=\frac{1}{K} \sum_{k=1}^{K} N_{b}(k), N_{a}=\frac{1}{K} \sum_{k=1}^{K} N_{a}(k), M_{b}=\frac{1}{K} \sum_{k=1}^{K} M_{b}(k), M_{a}=\frac{1}{K} \sum_{k=1}^{K} M_{a}(k)$, where $N_{b}(k)$, $N_{a}(k), M_{b}(k)$ and $M_{a}(k)$ denote the corresponding intervals in the k-th beat.

b) Each $\mathrm{P}$ wave in the training beats is forced to contain $N_{b}+N_{a}+1$ samples, namely $N_{b}$ samples before $\mathrm{P}$ wave peak and $N_{a}$ samples after $\mathrm{P}$ wave peak.

c) Each $\mathrm{T}$ wave in the training beats is forced to contain $M_{b}+M_{a}+1$ samples, namely $M_{b}$ samples before $\mathrm{T}$ wave peak and $M_{a}$ samples after T wave peak.

d) $\mu$ and $\sigma$ for $\mathrm{P}$ wave and $\mathrm{T}$ wave are estimated as:

$$
\begin{aligned}
& \mu_{X}(i)=\frac{1}{K} \sum_{k=1}^{K} X_{k}(i), \quad \sigma_{X}(i)=\frac{1}{K-1} \sqrt{\sum_{k=1}^{K}\left(X_{k}(i)-\mu_{X}(i)\right)^{2}}, \quad \text { for } i=1, \cdots, N \\
& \mu_{Y}(j)=\frac{1}{K} \sum_{k=1}^{K} Y_{k}(j), \quad \sigma_{Y}(j)=\frac{1}{K-1} \sqrt{\sum_{k=1}^{K}\left(Y_{k}(j)-\mu_{Y}(j)\right)^{2}}, \quad \text { for } j=1, \cdots, M
\end{aligned}
$$

e) $\beta_{X}(i) i=1, \cdots, N$ and $\beta_{Y}(i) i=1, \cdots, M$ are computed using Eq.(4). 
After the above procedures, the PDFs of $\mathrm{P}$ wave vector and $\mathrm{T}$ wave vector can be written as Eqs.(7) and (8), respectively.

$$
\begin{aligned}
& p_{X_{-} i}\left(x_{i}\right)=\frac{\omega\left(\beta_{X}(i)\right)}{\sigma_{X}(i)} \exp \left[-c\left(\beta_{X}(i)\right)\left|\frac{x_{i}-\mu_{X}(i)}{\sigma_{X}(i)}\right|^{2 /\left(1+\beta_{X}(i)\right)}\right], \quad i=1, \cdots, N \\
& p_{Y \_j}\left(y_{j}\right)=\frac{\omega\left(\beta_{Y}(j)\right)}{\sigma_{Y}(j)} \exp \left[-c\left(\beta_{Y}(j)\right)\left|\frac{y_{j}-\mu_{Y}(j)}{\sigma_{Y}(j)}\right|^{2 /\left(1+\beta_{Y}(j)\right)}\right], \quad j=1, \cdots, M
\end{aligned}
$$

\section{3. $T$ wave cancellation using MAP}

In traditional T wave cancellation methods, QRS-T template and QRS-T containing overlapping P wave are aligned with $R$ wave's peak when performing subtraction. Thus, the alignment of $T$ waves is poor, which would decrease the accuracy of $T$ wave cancellation methods. In this section, we use MAP method to estimate $\mathrm{T}$ wave template and the timing point of $\mathrm{T}$ wave peak simultaneously, and then $\mathrm{T}$ wave template and $\mathrm{T}$ wave containing overlapping $\mathrm{P}$ wave can be aligned with the estimated timing point of $\mathrm{T}$ wave peak, which would reduce the distortion of the extracted $\mathrm{P}$ wave.

$\mathrm{P}$ wave and $\mathrm{T}$ wave are induced by atrial activity and ventricular activity, respectively. Moreover, the $\mathrm{P}$ wave and $\mathrm{T}$ wave within the fused signal are from different cardiac beats. In view of these, they can be assumed to be mutually independent, and thus the joint PDF of $\mathrm{P}$ wave vector and $\mathrm{T}$ wave vector is the product of their own PDFs.

From the perspective of probability theory, $\mathrm{T}$ wave and the timing point of $\mathrm{T}$ wave peak can be estimated through calculating their MAP, that is

$$
\left[Y_{M A P}, \tilde{t}_{T}\right]=\arg \max \operatorname{Pr}\left(X, Y \mid t_{P}, t_{T}\right) \operatorname{Pr}\left(t_{P}, t_{T}\right)
$$

where $t_{P}$ and $t_{T}$ denote the timing points of $\mathrm{P}$ wave peak and T wave peak, respectively. $t_{P}$ and $t_{T}$ are assumed to be mutually independent and obey the uniform distribution within $\left[t_{P-L O W}, t_{P-H I G H}\right]$ and $\left[t_{T-L O W}, t_{T-H I G H}\right]$, respectively. When APC happens, there are no extreme changes in R-T interval and P-R interval comparing with the normal beat. In view of this, using the training samples mentioned in section 2.2, we determine $t_{P-L O W}=t_{R}(i)-\left(T_{P R}+30 \mathrm{~ms}\right), t_{P-H I G H}=t_{R}(i)-\left(T_{P R}-30 \mathrm{~ms}\right)$, $t_{T-L O W}=t_{R}(i-1)+\left(T_{R T}-60 \mathrm{~ms}\right)$ and $t_{T-H I G H}=t_{R}(i-1)+\left(T_{R T}+60 \mathrm{~ms}\right)$.

where $t_{R}(i)$ is the timing point of $\mathrm{R}$ wave in the APC beat, $t_{R}(i-1)$ is the timing point of $\mathrm{R}$ wave in the previous beat, $T_{P R}$ and $T_{R T}$ are the mean P-R interval (from $\mathrm{P}$ wave peak to $\mathrm{R}$ wave peak) and the mean R-T interval (from $\mathrm{R}$ wave peak to $\mathrm{T}$ wave peak) of the training beats, respectively. Considering the uniform distribution assumption of $t_{P}$ and $t_{T}$, the MAP estimation can be rewritten as:

$$
\left[Y_{M A P}, \tilde{t}_{T}\right]=\arg \max \operatorname{Pr}\left(X, Y \mid t_{P}, t_{T}\right)
$$

When only single-lead ECG signal is available (e.g., in some ECG monitor systems, the log form of Eq.10 is:

$$
\left[Y_{M A P}, \tilde{t}_{T}\right]=\arg \max _{X, Y, t_{P}, t_{T}} \log \left[\operatorname{Pr}\left(x_{1}, x_{2}, \cdots, x_{N}\right) \cdot \operatorname{Pr}\left(y_{1}, y_{2}, \cdots, y_{M}\right)\right]
$$


The specific forms of $\operatorname{Pr}\left(x_{1}, x_{2}, \cdots, x_{N}\right)$ and $\operatorname{Pr}\left(y_{1}, y_{2}, \cdots, y_{M}\right)$ depend on the overlap length and the relative location of $\mathrm{P}$ wave and $\mathrm{T}$ wave within the fused signals (see Figure 1).

Suppose the overlap length is $l(l>0) ; t_{P o n}$ and $t_{P o f f}$ represent the onset and offset timing points of $\mathrm{P}$ wave, respectively; $t_{T o n}$ and $t_{\text {Toff }}$ represent the onset and offset timing points of $\mathrm{T}$ wave, respectively; denotes the fused signal with $\mathrm{L}$ samples, from $\min \left[t_{P o n}, t_{T o n}\right]$ to $\max \left[t_{P o f f}, t_{T o f f}\right]$. Assuming the independence over time, two cases are considered:

a) When $t_{\text {Pon }} \geq t_{\text {Ton }}$ and $t_{\text {Poff }} \geq t_{\text {Toff }}$ (partially fused, see Figure 1(a)), we have:

$$
\begin{aligned}
& \operatorname{Pr}\left(x_{1}, x_{2}, \cdots, x_{N}\right)=\left(\prod_{i=1}^{l} p_{X_{-} i}\left(x_{i}\right)\right) \cdot\left(\prod_{i=l+1}^{N} p_{X_{-} i}(Z(L-N+i))\right) \\
& \operatorname{Pr}\left(y_{1}, y_{2}, \cdots, y_{M}\right)=\left(\prod_{i=1}^{M-l} p_{Y_{-} i}(Z(i))\right) \cdot\left(\prod_{i=M-l+1}^{M} p_{Y_{-} i}\left(Z(i)-x_{i-(M-l)}\right)\right)
\end{aligned}
$$

b) When $t_{P o n} \geq t_{T o n}$ and $t_{\text {Poff }}<t_{\text {Toff }}$ (completely fused, see Figure 1(b)), we have:

$$
\begin{aligned}
& \operatorname{Pr}\left(x_{1}, x_{2}, \cdots, x_{N}\right)= \prod_{i=1}^{N} p_{X_{-} i}\left(x_{i}\right) \\
& \operatorname{Pr}\left(y_{1}, y_{2}, \cdots, y_{M}\right)=\left(\prod_{i=1}^{t_{P o n}-t_{T o n}} p_{Y \_}(Z(i))\right) \cdot\left(\prod_{i=t_{P o n}-t_{T o n}+1}^{t_{P o n}-t_{T o n}+N} p_{Y \_}\left(Z(i)-x_{i-\left(t_{P o n}-t_{T o n}\right)}\right)\right) \\
& \cdot\left(\prod_{i=t_{\text {Pon }}-t_{T o n}+N+1}^{M} p_{Y \_i}(Z(i))\right)
\end{aligned}
$$

When multi-lead ECG recordings are available, the log form of Eq.(10) is expressed as:

$$
\left[Y_{M A P}, \tilde{t}_{T}\right]=\arg \max _{X, Y, t_{P}, t_{T}} L F
$$

where $L F=\sum_{j=1}^{J} \log \left[\operatorname{Pr}\left(x_{1}^{j}, x_{2}^{j}, \cdots, x_{N}^{j}\right) \cdot \operatorname{Pr}\left(y_{1}^{j}, y_{2}^{j}, \cdots, y_{M}^{j}\right)\right], J$ is the lead number, and $x_{1}^{j}, x_{2}^{j}, \cdots, x_{N}^{j}$ and $y_{1}^{j}, y_{2}^{j}, \cdots, y_{N}^{j}$ are the $\mathrm{P}$ wave vector and the $\mathrm{T}$ wave vector in the $j$-th lead ECG recording, respectively.

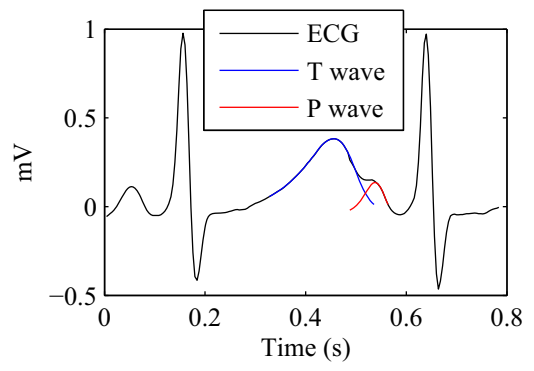

(a)

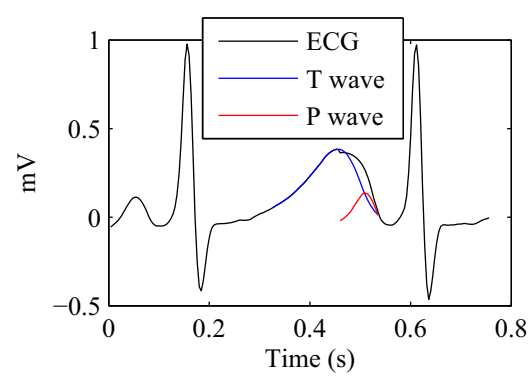

(b)

Fig. 1. Relative locations of $\mathrm{P}$ wave and $\mathrm{T}$ wave within the fused signal. 
After estimating $\mathrm{T}$ wave and the timing point of $\mathrm{T}$ wave peak, the estimated $\mathrm{T}$ wave is subtracted from the fused signal. Then, $\mathrm{P}$ wave can be detected using the traditional $\mathrm{P}$ wave detection procedures $[15,16]$. Here, the differential threshold-based detection method [15] is adopted to detect $\mathrm{P}$ wave in the remaining signal.

\section{Experiment results}

\subsection{Materials description}

In this section, the proposed method (denoted as 'OURS') is compared with Lin's T wave cancellation method [11] (denoted as 'LIN'). Both the synthetic and real fused signals are used to assess these two methods.

The synthetic fused signal is generated by manually shortening TP segments of normal ECG. Ten normal ECG recordings are chosen from MIT-BIH QT database [17]. Each of these ten recordings contains two-lead ECG signals with 11 bit resolution, sampled at a frequency of $250 \mathrm{~Hz}$. The fiducial points (onset, offset and peak) of $\mathrm{P}$ wave, $\mathrm{T}$ wave and QRS complex are all included in the annotation files. The first 100 beats of each normal recording are used to model the possibility distributions of $\mathrm{P}$ wave and $\mathrm{T}$ wave, and another 100 beats are used to generate the synthetic fused signals. Thus, there are totally 1000 synthetic fused beats.

Real ECG recordings with APC are chosen from MIT-BIH arrhythmia database. Here 7 recordings with APC are selected. Each recording includes two-lead ECG signals with 11 bit resolution, digitized at a sampling frequency of $360 \mathrm{~Hz}$.

\subsection{Performance assessment}

The normalized mean square error (NMSE) and cross-correlation index (CCI) between the extracted $\mathrm{P}$ wave and the original $\mathrm{P}$ wave are used to evaluate the methods' performance for the synthetic fused signal. NMSE and CCI are defined as:

$$
\begin{aligned}
& N M S E=\sqrt{\frac{\sum_{i=1}^{I}(S(i)-\tilde{S}(i))^{2}}{\sum_{i=1}^{I}(S(i))^{2}}} \\
& C C I=\frac{E[(S-E(S)) \cdot(\tilde{S}-E(\tilde{S}))]}{\sigma_{s} \sigma_{\tilde{S}}}
\end{aligned}
$$

where $S$ and $\tilde{S}$ are the original and the extracted $\mathrm{P}$ wave, respectively, $I$ is their length. $\sigma_{s}$ and $\sigma_{\tilde{S}}$ are standard deviations of $S$ and $\tilde{S}$, respectively. The smaller the NMSE is, the more accurate the method is. CCI is used to assess the morphology similarity of two signals. Its value would be 1 in the case of completely matching and would be 0 when two signals are completely different and unrelated.

It is hard to accurately evaluate the methods' performance for real fused signals, since the true $\mathrm{P}$ wave is unknown. Here we calculated CCI between the extracted $\mathrm{P}$ wave and the $\mathrm{P}$ wave of the preceding beat to determine whether the extracted $\mathrm{P}$ wave has the similar morphology with the $\mathrm{P}$ waves in normal beats. 


\subsection{Results for synthetic fused signals}

For each of the two-lead synthetic fused signals, the extracted $\mathrm{P}$ waves in the lead with more evident $\mathrm{P}$ waves are used for statistics. Table 1 summarizes NMSE and CCI at different overlapping lengths (ranging from $20 \mathrm{~ms}$ to $56 \mathrm{~ms}$ ). Figures 2 and 3 are the results. The proposed method performs much better than Lin's method in terms of NMSE and CCI, especially the mean NMSE of OURS is more than $10 \%$ less than that of LIN. From the partial enlarged view shown in Figures 2(b) and 3(b), we can see that the extracted $\mathrm{P}$ waves by our method are much closer to the original ones.

Table 1

NMSE and CCI for ten synthetic fused signals (mean \pm standard deviation)

\begin{tabular}{llll}
\hline Overlapping length $(\mathrm{ms})$ & Method & NMSE & CCI \\
\hline \multirow{2}{*}{30} & OURS & $0.07 \pm 0.01$ & $0.97 \pm 0.06$ \\
& LIN & $0.19 \pm 0.25$ & $0.93 \pm 0.09$ \\
& OURS & $0.14 \pm 0.28$ & $0.93 \pm 0.15$ \\
& LIN & $0.29 \pm 0.38$ & $0.90 \pm 0.13$ \\
44 & OURS & $0.27 \pm 0.64$ & $0.88 \pm 0.26$ \\
& LIN & $0.42 \pm 0.56$ & $0.87 \pm 0.18$ \\
56 & OURS & $0.40 \pm 0.94$ & $0.86 \pm 0.27$ \\
& LIN & $0.54 \pm 0.76$ & $0.85 \pm 0.18$ \\
\hline
\end{tabular}

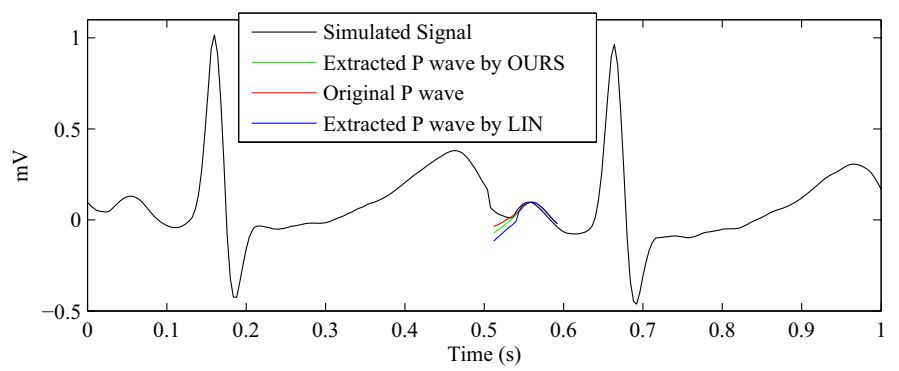

(a)

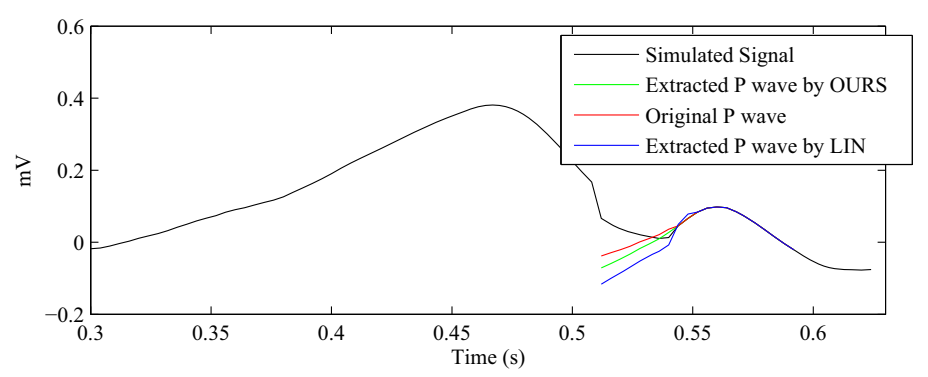

(b)

Fig. 2. Extraction of $P$ wave from the synthetic fused signals (overlapping length=32 ms). (a) ECG recording in the whole beat. (b) The partial enlarged view of (a). 


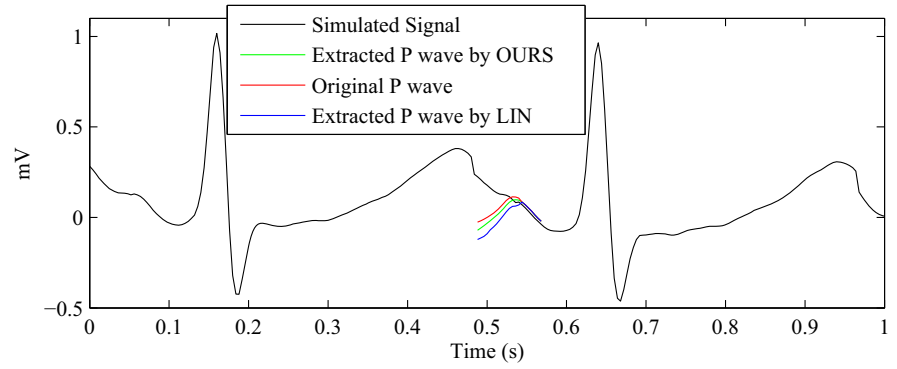

(a)

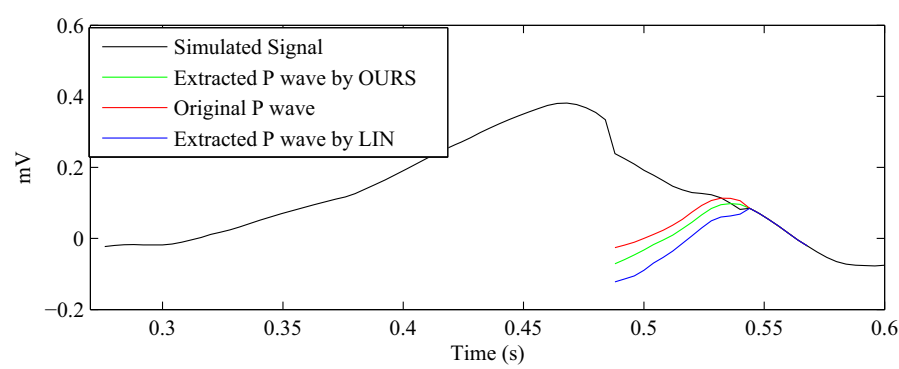

(b)

Fig. 3. Extraction of $\mathrm{P}$ wave from the synthetic fused signals (overlapping length=56 ms). (a) ECG recording in the whole beat. (b) The partial enlarged view of (a).

\subsection{Results for real fused signals}

In MIT-BIH arrhythmia database, there are no annotation files for the locations of $\mathrm{P}$ waves and $\mathrm{T}$ waves. Thus, we first use the differential threshold-based detection method [15] to annotate the locations of the non-overlapping $\mathrm{P}$ waves and T waves for each real ECG recording. After this, Lin's T wave cancellation method and the proposed method are applied to extract $\mathrm{P}$ wave from the fused signals.

Table 2 summarizes the performance of two methods for 7 real ECG recordings with APC. On the 8th row, the average of CCIs for each method is indicated. It can be seen that, in comparison with the extracted $\mathrm{P}$ wave using Lin's method, the extracted $\mathrm{P}$ waves using the proposed method are more similar to the non-overlapping $\mathrm{P}$ waves in morphology. Three examples are shown in Figures 4 and 5.

Table 2

CCI for real fused signals in MIT-BIH arrhythmia database (mean \pm standard deviation)

\begin{tabular}{llll}
\hline Recording name & APC number & OURS & LIN \\
\hline 100 & 33 & $0.77 \pm 0.32$ & $0.74 \pm 0.33$ \\
108 & 3 & $0.96 \pm 0.00$ & $0.85 \pm 0.06$ \\
202 & 30 & $0.54 \pm 0.38$ & $0.29 \pm 0.68$ \\
213 & 25 & $0.89 \pm 0.23$ & $0.25 \pm 0.77$ \\
220 & 73 & $0.83 \pm 0.17$ & $0.62 \pm 0.50$ \\
223 & 61 & $0.80 \pm 0.32$ & $0.37 \pm 0.67$ \\
233 & 6 & $0.80 \pm 0.40$ & $0.63 \pm 0.74$ \\
Average & 231 & $0.79 \pm 0.30$ & $0.50 \pm 0.61$ \\
\hline
\end{tabular}




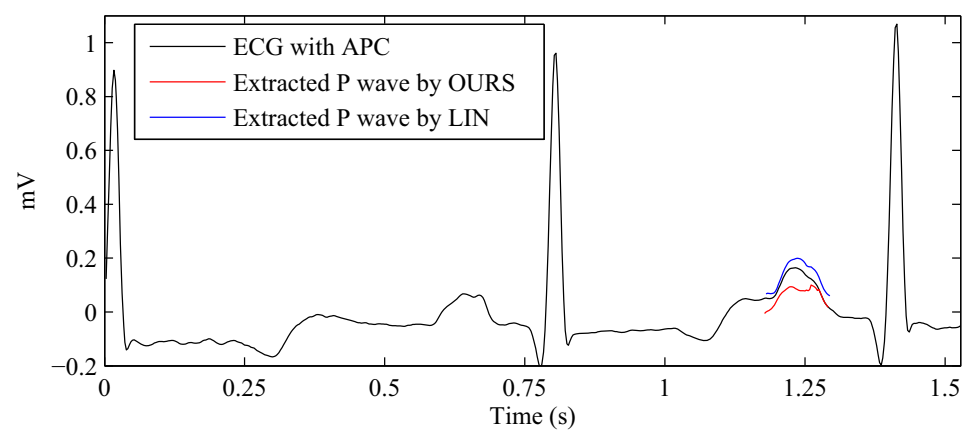

Fig. 4. Example 1 for extracting $P$ wave from real fused signals.

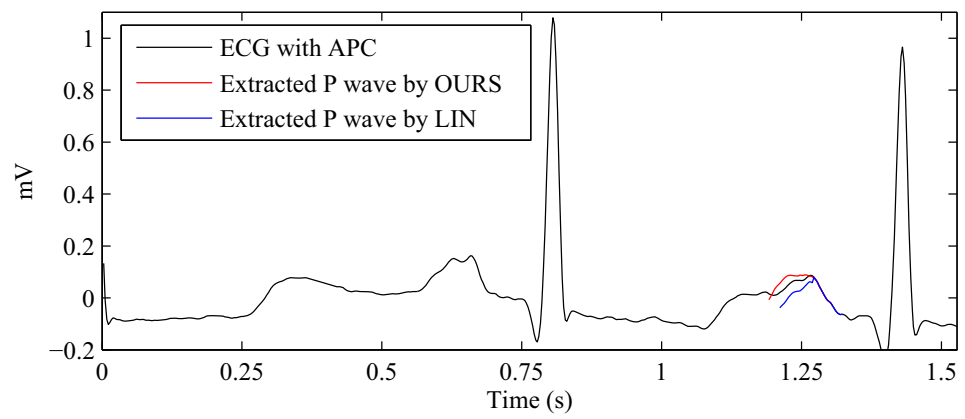

Fig. 5. Example 2 for extracting $P$ wave from real fused signals.

\section{Conclusions}

Extracting $\mathrm{P}$ wave from the fused signal is essential and inevitable for $\mathrm{P}$ wave measurement and further analysis. In this paper, we present a novel $\mathrm{T}$ wave cancellation method for $\mathrm{P}$ wave extraction based on MAP estimation. In comparison with traditional $\mathrm{T}$ wave cancellation methods, the proposed method has the ability to estimate $\mathrm{T}$ wave and the timing point of $\mathrm{T}$ wave peak simultaneously, which would make $\mathrm{T}$ wave cancellation more accurate. The experimental results confirmed the effectiveness of the proposed method, where the results for synthetic fused signals reveal that the proposed method is more accurate than the traditional $\mathrm{T}$ wave cancellation method.

In the experiments on real fused signals, because the true $\mathrm{P}$ wave within the fused signal is unknown, in this paper we just compared the extracted $\mathrm{P}$ waves using our method with the non-overlapping $\mathrm{P}$ waves. In future work, we will attempt to develop more reliable assessment indexes for the experiments on real fused signals. Moreover, we will make the further analysis for the extracted $\mathrm{P}$ waves in the future.

\section{References}

[1] F. Portet, P wave detector with pp rhythm tracking: evaluation in different arrhythmia contexts, Physiological Measurement 29 (2008), 141.

[2] J. P. Birkbeck, D. B. Wilson, M. A. Hall and D. G. Meyers, P-wave morphology correlation with left atrial volumes assessed by 2-dimensional echocardiography, Journal of Electrocardiology 39 (2006), 225-229. 
[3] D. Kitkungvan and D. H. Spodick, Interatrial block: is it time for more attention?, Journal of Electrocardiology 42 (2009), $687-692$

[4] J. Vağgaisăğanen and P. Korhonen, Effective p-wave detection for analyzing long-term changes in atrial activation, Journal of Electrocardiology 40 (2007), S35.

[5] G. Carrault, M.-O. Cordier, R. Quiniou and F. Wang, Temporal abstraction and inductive logic programming for arrhythmia recognition from electrocardiograms, Artificial Intelligence in Medicine 28 (2003), 231-263.

[6] C. Kolb, S. Ną̆urnberger, G. Ndrepepa, B. Zrenner, A. Schą̆gomig and C. Schmitt, Modes of initiation of paroxysmal atrial fibrillation from analysis of spontaneously occurring episodes using a 12-lead holter monitoring system, The American Journal of Cardiology 88 (2001), 853-857.

[7] J.Willems, Recommendations for measurement standards in quantitative electrocardiography, European Heart Journal 6 (1985), 815-825.

[8] A. Cabasson, O. Meste, G. Blain and S. Bermon, A new modeling of the overlapping $t$ wave for the efficient estimation of the pr intervals during exercise and recovery, Engineering in Medicine and Biology Society, 29th Annual International Conference of the IEEE, Lyon, France, 2007, 616-619.

[9] A. Cabasson, O. Meste, G. Blain and S. Bermon, A time delay estimation technique for overlapping signals in electrocardiograms, Proceedings of EUSIPCO 16 (2008).

[10] A. Cabasson, O. Meste, G. Blain and S. Bermon, Quantifying the pr interval pattern during dynamic exercise and recovery, Biomedical Engineering, IEEE Transactions on 56 (2009), 2675-2683.

[11] B. Lin, P. J. Wang, S. Mahapatra, M. Homoud, M. Link, N. Estes and A. Al-Ahmad, Extraction of buried p waves from printed electrocardiograms, Annals of Noninvasive Electrocardiology 10 (2005), 142-145.

[12] H. Dai, S. Jiang and Y. Li, Atrial activity extraction from single lead ECG recordings: evaluation of two novel methods, Computers in Biology \& Medicine 43 (2013), 176-183.

[13] B. G and T. G, Baysian Inference in Statistical Analysis, John Wiley and Sons, New York, 1973.

[14] T. W. Lee and M. S. Lewicki, The generalized gaussian mixture model using ica, International Workshop on Independent Component Analysis, Helsinki, Finland, 2000, 239-244.

[15] P. Laguna, R. Jane and P. Caminal, Automatic detection of wave boundaries in multilead ecg signals: Validation with the cse database, Computers and Biomedical Research 27 (1994), 45-60.

[16] C. Li, C. Zheng and C. Tai, Detection of ecg characteristic points using wavelet transforms, Biomedical Engineering, IEEE Transactions on 42 (1995), 21-28.

[17] P. Laguna, R. G. Mark, A. Goldberg and G. B. Moody, A database for evaluation of algorithms for measurement of qt and other waveform intervals in the ecg, Computers in Cardiology 24 (1997), 673-676. 